\title{
ON THE APPLICATION OF SPECTRAL UNMIXING FOR NOISE REDUCTION
}

\author{
Daniele Cerra, Rupert Müller, Jakub Bieniarz, and Peter Reinartz.
}

\author{
German Aerospace Center(DLR) \\ Remote Sensing Technology Institute (IMF) \\ 82234 Wessling, Germany
}

\begin{abstract}
Spectral unmixing aims at decomposing each image element of a hyperspectral scene in signals typically related to pure materials. This paper gives an added value to the results of this process by proposing Unmixing-based Denoising (UBD), a supervised methodology to recover bands characterized by a low Signal-to-Noise Ratio in a hyperspectral scene. In the first step of UBD, an unmixing procedure is carried out using a set of reference spectra which are noisefree, as they are averaged over areas for which ground truth is available. Results are inferred into the pixelwise reconstruction of a given band, expressed as a linear combination of the values of the reference spectra in that band, ignoring the residual vector which is mainly characterized by undesired atmospheric influences and sensor-induced noise. The reconstructed images exhibit both high visual quality and reduced spectral distortions.
\end{abstract}

Index Terms - Spectral unmixing, denoising, image restoration, hyperspectral images.

\section{INTRODUCTION}

Traditional denoising techniques employed in hyperspectral image processing exploit the strong redundancies typical of these data. A popular choice is represented by dimensionality reduction techniques, which project the data onto a subspace where meaningful information is preserved, removing noise and some high frequencies in the process [1]. Among others, ad hoc methods have been proposed which threshold wavelet coefficients [2], or enforce regularities in noisy bands by minimizing the Total Variation in the data and exploiting interband correlations $[3,4]$. In all cases, each value in a given noisy band is reconstructed as a linear combination of its values in neighbouring bands, or as a (often non-linear) transformation which takes into account neighbouring image elements in two or three dimensions.

While the experiments in the aforementioned works are derived from a statistical analysis of the data, this paper proposes to use Unmixing-based Denoising (UBD), a methodology resulting from considering the physical properties of any hyperspectral image element. This is assumed to be a mixture of a number of materials smaller than the original data dimensionality, and can be effectively described relying on spectral unmixing methods. The challenging process of spectral unmixing aims at providing accurate information on a hyperspectral signature at sub-pixel level, by quantitatively decomposing each image element in signals typically related to macroscopically pure materials, or endmembers. In the linear mixture model, each spectrum is approximated by a linear combination of the endmembers plus a residual vector. The latter is the sum of the contributions of noise, subtle variations within the reference materials, and errors in the adopted unmixing model [5]. If the set of endmembers is complete and well represents the scene at hand, and if the reference spectra exhibit negligible noise influences, the residual vector will mostly result from atmospheric interferences and instrumentinduced noise, and can be ignored in the reconstruction of each single spectrum. Thus, spectral unmixing finds through UBD a new field of application, as the results of unmixing are used as prior knowledge to reconstruct each pixel in a given band of a hyperspectral image as a linear combination of the values of the reference spectra in that particular band. As such values would not be reliable for a band with low Signal-toNoise Ratio, the reference spectra are averaged over an area of interest to greatly reduce the noise influence in the input to the reconstruction.

Results on real data are promising and show that the proposed method would be particularly effective on bands with a low Signal-to-Noise Ratio (SNR). This would allow exploiting the spectral information contained in these bands, which are often discarded in a preprocessing step common to most practical applications.

The remainder of the paper is organized as follows. Section 2 illustrates the proposed denoising methodology based on spectral unmixing, Section 3 reports experimental results, and Section 4 contains concluding remarks.

\section{UNMIXING-BASED DENOISING}

Given a hyperspectral image element $m$ with $p$ bands, and a training dataset containing $n$ samples from each of $k$ classes, with $k<p$, the Unmixing-based Denoising (UBD) is a simple procedure which can be described as follows. 
Firstly, a set of reference spectral signatures is defined as $A=\left\{x_{1}, \ldots, x_{i}, \ldots, x_{k}\right\}$, where $x_{i}$ is the average of the $n$ spectra belonging to class $i$. Considering the mean value for a given reference spectrum reduces the presence of noise to a minimum, if each class is spectrally homogeneous. It must be remarked that no assumption on the purity of the reference spectra is made. Then, any unmixing procedure can be employed to decompose the signal in a combination of the reference spectra. If we assume this to be linear, we have:

$$
m=\sum_{i=1}^{k} x_{i} s_{i}+r,
$$

where $s_{i}$ is the fraction or abundance of the reference spectrum $i$ in $m$, and $r$ the residual vector. The latter is mostly composed by the contributions related to materials not present in $A$, subtle variations of one or more materials in $A$, atmospheric interferences, and instrument-induced noise. If the spectra in $A$ are noise-free and represent well the classes of interest, we expect the last two terms to be predominant in the residual vector for bands with low SNR, and we can derive a reconstruction $\hat{m}$ as:

$$
\hat{m}=\sum_{i=1}^{k} x_{i} s_{i},
$$

ignoring $r$, and along with it most of the noise affecting $m$. The described procedure is based on the assumption that if the contributions to the radiation reflected from a resolution cell are known, the value of noisy bands in that area can be derived by a combination of the average values characterizing each component in that spectral range. The proposed method is supervised, as it needs as input a set of spectra that well characterize the scene, and is carried out independently for each pixel. As a certain homogeneity of the classes of interest is assumed, the method is expected to perform better on natural scenes where man-made objects (usually having a higher variability) are not prevalent.

\section{EXPERIMENTS}

In the following experiments we choose Non-negative Least Squares (NNLS) as unmixing algorithm [5]. Unconstrained Least Squares and first attempts at using sparse reconstruction tools did not yield satisfactory results.

\subsection{Cuprite dataset}

We analyze the popular AVIRIS Cuprite hyperspectral scene, of size $512 \times 217$ pixels with 224 bands in the spectral range $0.38-2.5 \mu \mathrm{m}$. The last band centered at $2.5 \mu \mathrm{m}$ is severely affected by noise (Fig.1). A geological map [6] has been used to manually select 15 reference spectra belonging to different minerals, averaged in areas of size $5 \times 5$ in the image. The results for UBD applied as in Eq. (2) using the described
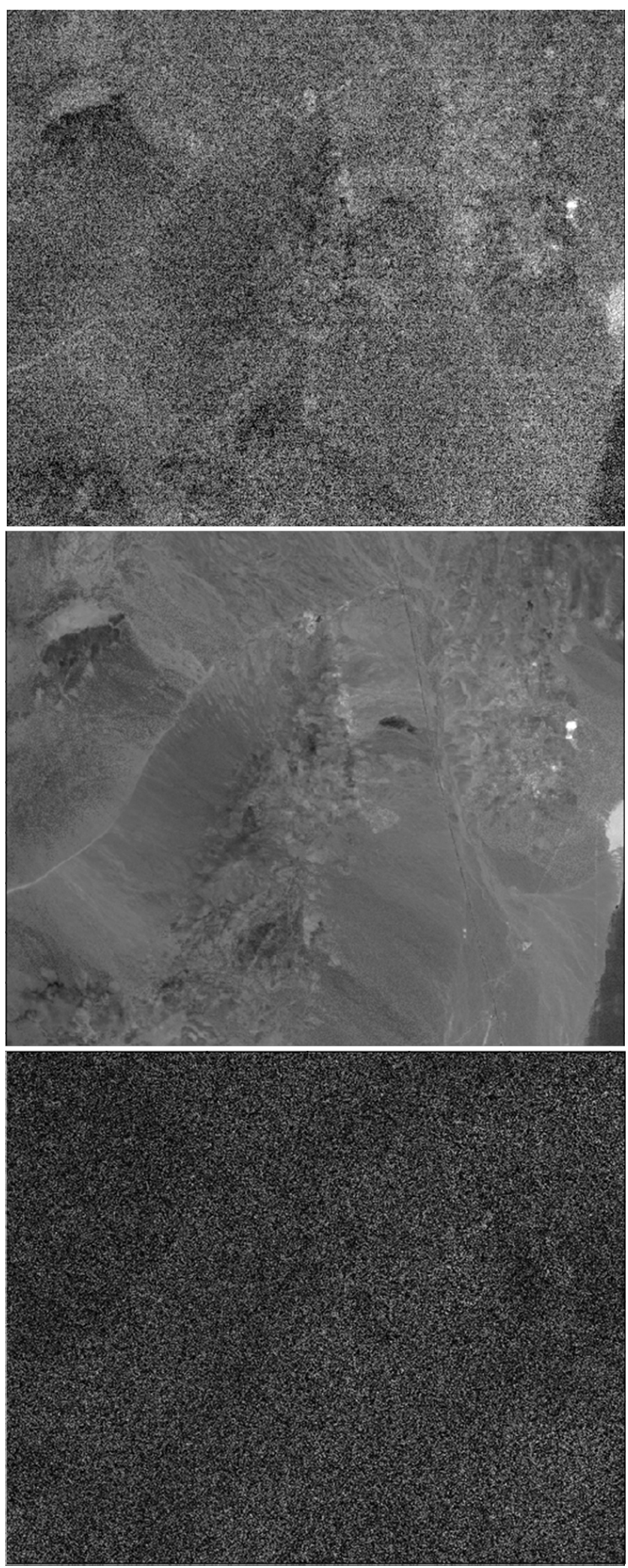

Fig. 1. Cuprite band 224, from top down: original image, UBD results, and stretched difference between the two images. It is not easy to detect in the bottom image features denoting loss of informational content due to the UBD process. 


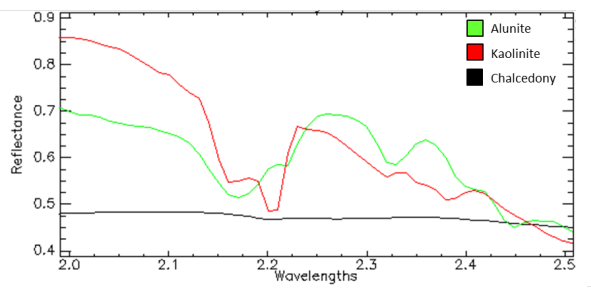

(a)

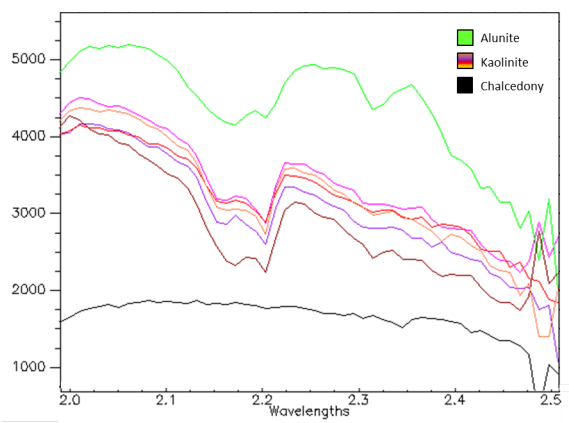

(b)

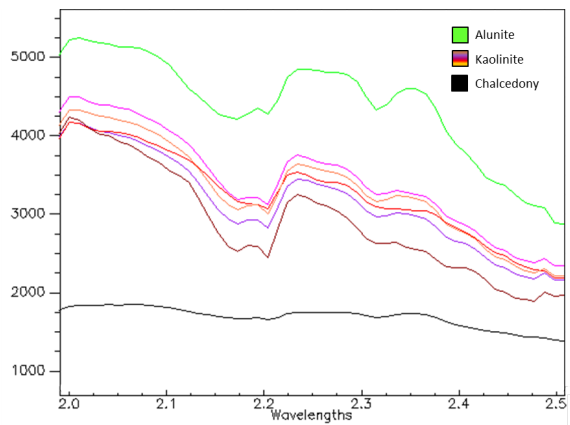

(c)

Fig. 2. From left to right: (a) laboratory spectra; (b) spectra related to the materials in (a) identified in the Cuprite dataset; (c) spectra from (b) reconstructed through UBD.

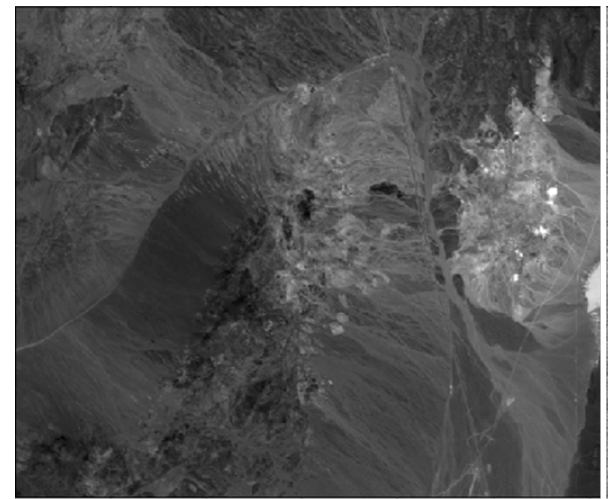

(a)

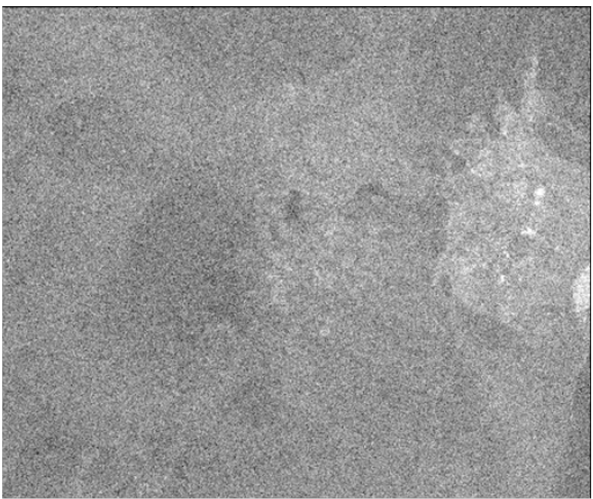

(b)

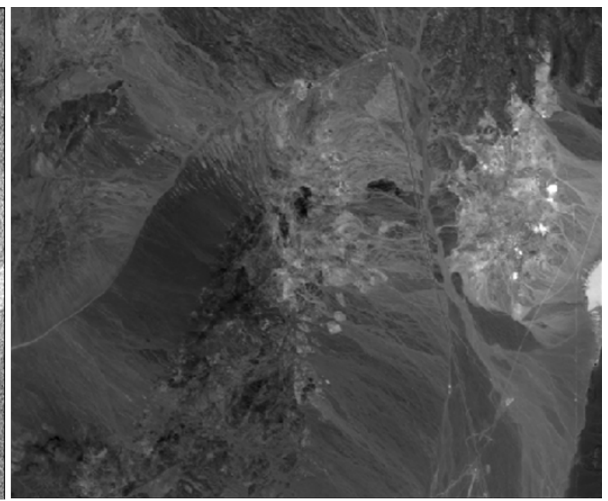

(c)

Fig. 3. Cuprite denoising experiment, from left to right: (a) band 42 from the dataset ( $750 \mathrm{~nm}$ ); (b) band 42 severely corrupted by additive white Gaussian noise with $\sigma^{2}=10^{6}$; (c) image (b) denoised through UBD.

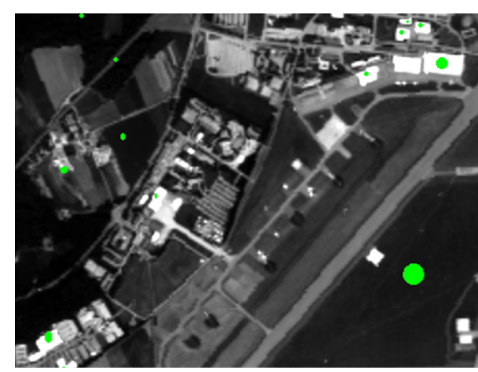

(a)

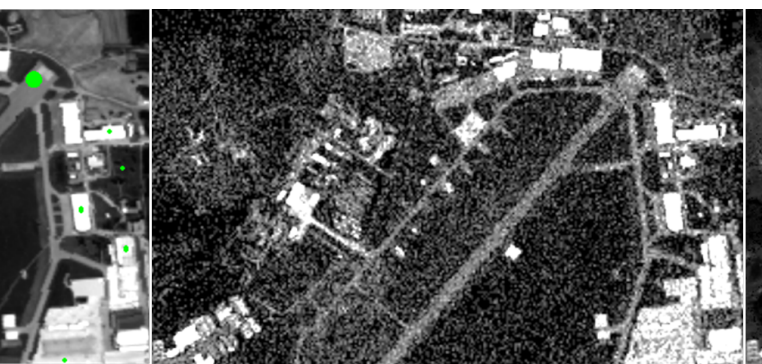

(b)

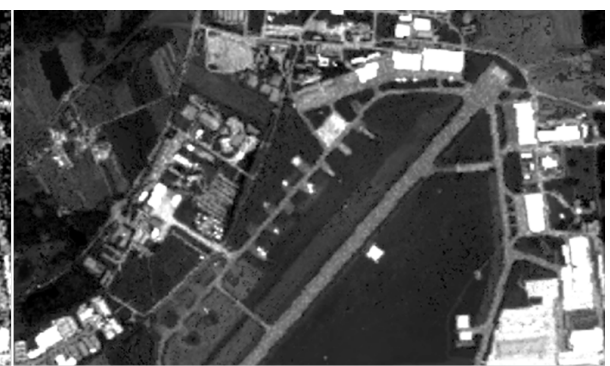

(c)

Fig. 4. UBD results on a HyMAP subscene acquired over DLR facilities, Oberpfaffenhofen, Germany. From left to right: (a) band 15 with regions of interest selected for the following denoising procedure; (b) band 1 from the original dataset (440 $\mathrm{nm}$ ); (c) band 1 denoised.

spectral library as input are reported in Fig. 1, along with the image of the difference between original and denoised band suggesting that most of the removed signal is random noise.

We analyze the spectral range 2-2.5 $\mu \mathrm{m}$ as in [7] to check the spectral integrity of the image elements in this range and their behaviour across noisy bands. Fig. 2 (a) reports the spectral signatures of 3 materials which are known to be part of the scene (alunite, kaolinite, and chalcedony), resampled to match the spectra in the data. Fig. 2 (b) contains the spectra related to some pixels which contain a high concentration 
of the mentioned materials [6], while Fig. 2 (c) shows the same spectra after UBD, which in the lowest frequencies are considerably smoother and match well the laboratory spectra, while the characteristics of single image elements are mostly kept in the results.

In a second experiment we severely corrupt band 42 of the dataset, centered at $750 \mathrm{~nm}$, with additive white gaussian noise (AWGN) having variance $\sigma^{2}=10^{6}$. The original and noisy bands along with the results of UBD using as input reference spectra collected from the corrupted image are reported in Fig. 3. It should be remarked that results in Fig. 3 (c) are obtained pixelwise using values computed from the noisy band in Fig. 3 (b) only, and enforcing the spectral abundances computed using the full image.

\subsection{HyMAP dataset}

A second experiment is carried out on a HyMAP dataset acquired over the DLR facilities in Oberpfaffenhofen, Germany, of size $300 \times 500$ and containing 128 spectral bands. One average reference spectrum has been collected from each of the 18 areas marked in Fig.4 (a). We applied UBD to the full dataset and report in Fig. 4 (b-c) the denoising results for the first band of the dataset, centered at $440 \mathrm{~nm}$. The noise is greatly reduced, although it is still visible in some areas (specially on the runway), while relevant information is well preserved. The algorithm introduces a low overall distortion across all bands, as the average spectral angle value between the original and the denoised image results equal to $1.29 \times 10^{-3}$.

\section{CONCLUSIONS}

Unmixing-based Denoising (UBD) is a supervised methodology for the recovery of bands characterized by a low Signalto-Noise Ratio (SNR) in a hyperspectral scene. UBD reconstructs any pixel in a given band as a linear combination of reference spectra belonging to materials present in the scene, which have negligible noise influences as they are averaged over areas for which ground truth is available. As the residual vector from the unmixing process is mostly composed by contributions of uninteresting materials, unwanted atmospheric influences and sensor-induced noise, this is ignored in the reconstruction process. Experiments on real data suggest that this method could be used to retrieve usable spectral information from bands which are usually discarded in practical applications. An example is represented by applications to natural waters, where the coloured Dissolved Organic Matter (CDOM) could be better estimated by employing directly the spectral information at the edge between visible and Near Ultraviolet frequencies, which is typically noisy [8]. A drawback of the method is that it requires as input a reasonably large amount of pixels to derive each reference spectrum, in order to have a meaningful mean value robust to noise and local variations. The reference spectra selection step may be completely replaced in the future by estimating the virtual dimensionality of a hyperspectral scene and then selecting the centroids of a relevant number of clusters after an unsupervised clustering step. Furthermore, as the proposed algorithm operates pixelwise it could be further improved by better exploiting spatial information: the mentioned Total Variation (TV) model could be applied not for direct denoising of the data, but for improving the unmixing procedure which constitutes the first step for UBD [9].

\section{REFERENCES}

[1] N. Renard, S. Bourennane, and J. Blanc-Talon, "Denoising and dimensionality reduction using multilinear tools for hyperspectral images," IEEE Geoscience and Remote Sensing Letters, vol. 5, no. 2, pp. 138 -142, april 2008.

[2] G. Chen and S. Qian, "Denoising of hyperspectral imagery using principal component analysis and wavelet shrinkage," IEEE Transactions on Geoscience and Remote Sensing, vol. 49, no. 3, pp. 973 -980, march 2011.

[3] Q. Yuan, L. Zhang, and H. Shen, "Hyperspectral image denoising employing a spectral-spatial adaptive total variation model," IEEE Transactions on Geoscience and Remote Sensing, vol. 50, no. 10, pp. 3660 -3677, oct. 2012.

[4] H. Zhang, "Hyperspectral image denoising with cubic total variation model," ISPRS Annals of Photogrammetry, Remote Sensing and Spatial Information Sciences, vol. I7, pp. 95-98, 2012.

[5] J. M. Bioucas-Dias, A. Plaza, N. Dobigeon, M. Parente, Q. Du, P. Gader, and J. Chanussot, "Hyperspectral unmixing overview: Geometrical, statistical, and sparse regression-based approaches," IEEE Journal of Selected Topics in Applied Earth Observations and Remote Sensing, vol. 5, no. 2, pp. 354-379, 2012.

[6] USGS Spectroscopy Lab, "Cuprite geological map," http://speclab.cr.usgs.gov/cuprite95.tgif.2.2um_map.gif.

[7] M. Parente and A. Zymnis, "Statistical clustering and mineral spectral unmixing in aviris hyperspectral image of cuprite, nv," Tech. Rep., CS229, 2005.

[8] T. Kutser, D. C. Pierson, K. Y. Kallio, A. Reinart, and S. Sobek, "Mapping lake cdom by satellite remote sensing," Remote Sensing of Environment, vol. 94, no. 4, pp. 535 - 540, 2005.

[9] M.-D. Iordache, J.M. Bioucas-Dias, and A. Plaza, “Total variation spatial regularization for sparse hyperspectral unmixing," IEEE Transactions on Geoscience and Remote Sensing, vol. 50, no. 11, pp. $4484-4502$, nov. 2012. 